ARTICLE

Received 9 Dec 2016 | Accepted 13 Jan 2017 | Published 24 Feb 2017

DOI: $10.1038 /$ ncomms14619

OPEN

\title{
Direct experimental determination of the topological winding number of skyrmions in $\mathrm{Cu}_{2} \mathrm{OSeO}_{3}$
}

S.L. Zhang ${ }^{1}$, G. van der $\operatorname{Laan}^{2} \&$ T. Hesjedal ${ }^{1}$

The mathematical concept of topology has brought about significant advantages that allow for a fundamental understanding of the underlying physics of a system. In magnetism, the topology of spin order manifests itself in the topological winding number which plays a pivotal role for the determination of the emergent properties of a system. However, the direct experimental determination of the topological winding number of a magnetically ordered system remains elusive. Here, we present a direct relationship between the topological winding number of the spin texture and the polarized resonant $\mathrm{X}$-ray scattering process. This relationship provides a one-to-one correspondence between the measured scattering signal and the winding number. We demonstrate that the exact topological quantities of the skyrmion material $\mathrm{Cu}_{2} \mathrm{OSeO}_{3}$ can be directly experimentally determined this way. This technique has the potential to be applicable to a wide range of materials, allowing for a direct determination of their topological properties. 
n a many-body system, a local order parameter can be assigned to individual entities, and by considering interactions among them, emergent phases and novel physical properties may evolve. The possible values of the order parameters constitute the order parameter space, which can be described in the framework of topology 1,2 . In magnetism, the spins are the elementary entities, and the order parameter is the magnetization vector $\mathbf{m}$. Its magnitude can be taken as a constant, that is, its three components satisfy $m_{1}^{2}+m_{2}^{2}+m_{3}^{2}=M_{S}^{2}$, where $M_{\mathrm{S}}$ is the saturation magnetization. Therefore, the order parameter space is the surface of a three-dimensional unit sphere, which is described by the homotopy group $\pi_{2}\left(S^{2}\right)$ for a two-dimensional physical space $(x, y)$ (ref. 2). Different homotopy classes with distinct topological properties can be quantified based on the winding number $N$, which is an integer that counts the number of times the physical space fully covers the order parameter space ${ }^{3}$. It is defined as

$$
N=\frac{1}{4 \pi} \int\left(\frac{\partial \mathbf{m}}{\partial x} \times \frac{\partial \mathbf{m}}{\partial y}\right) \cdot \mathbf{m} \mathrm{d} x \mathrm{~d} y .
$$

Recently, it has been demonstrated that non-centrosymmetric helimagnetic materials carry $N=1$ magnetic skyrmions ${ }^{4-6}$, which leads to emergent phenomena, including novel magnetoelectrical transport effects (that is, the topological Hall effect $^{7-9}$, skyrmion motion induced by ultralow current densities ${ }^{10-13}$, and emergent electromagnetic fields ${ }^{14-16}$ ), as well as new spin dynamic properties ${ }^{17-19}$. Utilizing this nontrivial topological order, advanced spintronics applications have been devised ${ }^{20-24}$. More recently, several candidates with $N=2$ have also been discovered $^{25,26}$, suggesting that other elements from the $\pi_{2}\left(S^{2}\right)$ group may exist in nature as well.

While the significance of the topological properties of ordered systems is being recognized more and more, the experimental determination of the winding number for spin-ordered media remains challenging. Commonly, the winding number is determined by comparing a microscopic image of the magnetization state with theoretical model calculations, making it a rather indirect process that has no unique answer ${ }^{21-24,27-29}$. Most importantly, the established magnetic imaging techniques only give a partial picture of the local magnetization vector, as they are both limited in three-dimensional sensitivity and lateral resolution. For example, Lorentz transmission electron microscopy (LTEM) has been the most common technique which is used to infer the topological winding number from a magnetization map ${ }^{27}$. In most of the LTEM experiments, the magnetization configuration is obtained via an indirect transport-of-intensity equation simulation process, and, most importantly, the information of the out-of-plane spin component is missing ${ }^{27}$. Consequently, LTEM is not a direct experimental method $^{30}$ to determine the topological winding number (see Supplementary Note 3 for a detailed discussion). On the other hand, the presence of skyrmions leads to measurable signals in electric transport, that is, the topological Hall effect ${ }^{9}$. Nevertheless, other non-collinear magnetic structures, which are not related to skyrmions, can also give rise to a measurable topological Hall effect ${ }^{7,31}$, rendering transport measurements less ideal for the unambiguous determination of topological properties.

Here, we show that the winding number $N$ can be unambiguously identified by utilizing the sensitivity of the light polarization to the magnetic order at resonant elastic $\mathrm{X}$-ray scattering (REXS) condition, referred as polarizationdependent REXS.

\section{Results}

Representation of a skyrmion with winding number $N$. A general magnetic skyrmion structure can be obtained by mapping the order parameter space to the physical space, described in the two-dimensional polar coordinates $\rho$ (radial coordinate) and $\Psi$ (azimuthal angle), in the following way $^{4,32}$ :

$$
\begin{aligned}
& m_{1}=M_{\mathrm{S}} \sin \Theta(\rho) \cos [N(\Psi+\chi)], \\
& m_{2}=M_{\mathrm{S}} \sin \Theta(\rho) \sin [N(\Psi+\chi)], \\
& m_{3}=M_{\mathrm{S}} \lambda \cos \Theta(\rho),
\end{aligned}
$$

where the boundary conditions are defined such that the magnetization points up in the centre of the two-dimensional physical space and down at the boundary. The function $\Theta(\rho)$ describes the radial profile of the out-of-plane component of the magnetization, starting from the centre and extending to the boundary; $\chi$ is the helicity, defined in the range of $(-\pi, \pi]$; and $\lambda$ takes the values of \pm 1 , describing the polarity of the skyrmion ${ }^{4}$. For example, an $N=1$ skyrmion appears as a vortex-like texture. If the core magnetization points up (that is, $\lambda=1$ ), a $\chi=-\pi / 2$ skyrmion has a clockwise rotation sense when viewing from the top. The entire texture thus carries negative chirality, defined by $C=\operatorname{sgn}(\lambda \chi)$. Analogously, a $\chi=\pi / 2$ skyrmion carries positive chirality. On the other hand, for $\chi=0$ and $\chi=\pi$ skyrmions, so-called Néel-type skyrmions, there is no chirality present ${ }^{33}$.

Another, more illustrative way to interpret equation (2), is to construct an $\mathrm{N}$-skyrmion texture by assigning a one-dimensional helix spin profile to a radial chain in physical space, and by repeating the process for all azimuthal angles $\Psi$, in the range from $0^{\circ}$ to $360^{\circ}$, thereby mapping out the entire two-dimensional physical space. This concept is illustrated in Fig. 1a-c. Starting from the line for $\Psi=0^{\circ}$ that is parallel to $x$ axis, a standard harmonic helix structure is assigned. Subsequent $\Psi$ angles get a helix assigned that is rotated by $N \Psi$ from $\Psi=0^{\circ}$. As as result, when the two-dimensional physical space is fully sampled, the order parameter space will have been mapped out $N$ times. The exact structure of such harmonic helices does not affect the topological properties of the system, nor our measurement principle, as will be shown below. Using this one-dimensional helix approximation, an analytical solution for the polarizationdependent REXS process can be obtained that is explicit in $N$.

Topology determination principle. The measurement geometry for determining $N$ is illustrated in Fig. 1d. The incident and scattered X-ray wavevectors are denoted as $\mathbf{k}_{\mathrm{i}}$ and $\mathbf{k}_{\mathrm{s}}$, with the incident angle $\alpha$, which satisfies the diffraction condition $\mathbf{Q}=\mathbf{k}_{\mathrm{s}}-\mathbf{k}_{\mathbf{i}}$. The incident $\mathrm{X}$-rays can be linearly polarized with the polarization angle $\beta$. We define $\beta=0^{\circ}$ corresponding to $\sigma$-polarization, while $\beta=90^{\circ}$ corresponds to $\pi$-polarization. Alternatively, the light can be circularly polarized. Here, we define the circular dichroism (CD) signal as the difference of the scattering cross-sections for left-circularly and rightcircularly polarized incident light (at the same diffraction condition).

We demonstrate our new experimental principle for the determination of $\mathrm{N}$ on the magnetic skyrmion system $\mathrm{Cu}_{2} \mathrm{OSeO}_{3}$. This material carries an incommensurate, hexagonal lattice with an $N \neq 0$ topological entity motif ${ }^{34-36}$. The modulation wavevector is $\sim 0.0158$ r.l.u., and the motif's periodic lattice lies in the $x-y$-plane when the required magnetic field is along the $z$ direction $^{37}$ (see Fig. 1). Using the one-dimensional helix approximation construction, an analytical form of the resonant magnetic diffraction cross-section $I$ and the $C D$ cross-section $I_{\mathrm{CD}}$ can be derived as a function of 
a

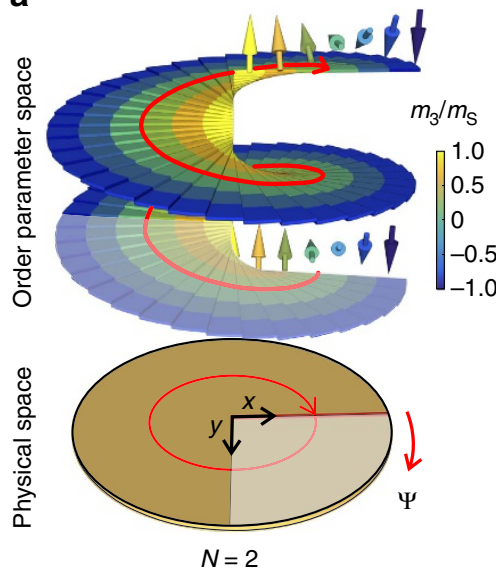

b

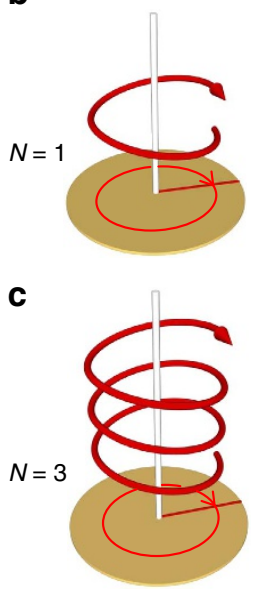

d

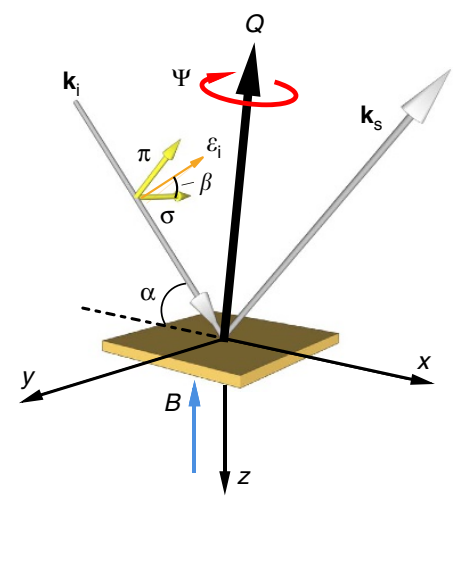

Figure 1 | Concept of winding number and experimental setup. (a) For classical spins in two-dimensional space, the spin configuration that carries a winding number of $N$, as described by equation (1), can be equivalently constructed by mapping the two-dimensional physical space using one-dimensional helices. In order parameter space, the one-dimensional helices are rotated azimuthally by $N \Psi$ from the base position (see, for example, bottom spin helix for $\Psi=0^{\circ}$ ), and projected onto physical space at the azimuthal position $\Psi$. The helices are stacked up in order parameter space for illustrative purposes following the red helical guideline. The example shows the situation for $N=2$ where the order parameter space maps the physical space twice. The shaded quarter-circle in physical space (below) corresponds to a covered half-circle in order parameter space (above). Simplified plots for the $N=1$ and $N=3$ cases are shown in $\mathbf{b}, \mathbf{c}$, respectively. (d) When $\Psi$ covers the range from $0^{\circ}$ to $360^{\circ}$, the scattered intensity will exhibit a periodicity that only depends on N. Both circularly or linearly polarized incident light is used, allowing for two measurement strategies: CD plots and PAMs. In both cases, the diffraction condition is met for the wavevector $\mathbf{Q}$, which contains the topological motif's modulation wavevector for different azimuthal angles $\Psi$. The polarization angle $\beta$ of the incident light polarization vector $\epsilon_{\mathrm{i}}$ is defined with respect to $\sigma$-polarization ( $\beta=0^{\circ} ; \beta=90^{\circ}$ corresponds to $\pi$-polarization).

$N$ (see Methods for the derivation)

$$
\begin{aligned}
I(\beta, \Psi, N)= & Y \sin ^{2} \alpha+Y \cos ^{2} \alpha \sin ^{2}\left(N \Psi+\Phi_{2}\right) \\
& +4 Y \cos ^{2} \alpha \sin ^{2} \alpha \cos ^{2}\left(N \Psi+\Phi_{2}\right) \sin ^{2} \beta \\
& -Y \cos ^{2} \alpha \sin \alpha \sin \left(2 N \Psi+2 \Phi_{2}\right) \sin 2 \beta,
\end{aligned}
$$

and

$$
I_{\mathrm{CD}}(\Psi, N)= \begin{cases}\mathcal{C} \cos \left(N \Psi+\Phi_{1}\right) & ; \text { for } N \text { odd }, \\ 0 & ; \text { for } N \text { even }\end{cases}
$$

where $\mathcal{C}$ and $Y$ are constants. The arbitrary phase parameters $\Phi_{1}$ and $\Phi_{2}$ can be chosen to adapt to other spin configurations with the same winding number, however, which deviate from the 'standard' configuration as constructed in Fig. 1a. These two relationships can be interpreted in the following way, which form the core of our experimental method for the determination of the winding number: In case of an odd winding number, $N$ equals to the periodicity of the CD signal, while $\Psi$ covers the full range from $0^{\circ}$ to $360^{\circ} . N$ is also equal to half the number of peaks in the polarization-azimuthal map (PAM; see below). For an even winding number, no $\mathrm{CD}$ signal is observed. The case of non-integer winding numbers is discussed in Supplementary Note 2.

Numerical results. Figure $2 \mathrm{e}-\mathrm{h}$ shows the numerical calculation results for the $\mathrm{CD}$ cross-section for different topological spin motifs. Equation (4) can be represented by the CD amplitude as a function of a closed-loop in reciprocal space. Each reciprocal space point $\left(q_{x}^{\text {diff }}, q_{y}^{\text {diff }}\right)$ on the loop (shown in red) corresponds to one azimuthal angle at which the diffraction condition for the modulation wavevector for $\Psi$ is met. Therefore, according to equation (4), the CD intensity varies as a function of $\Psi$, with a periodicity that is equal to $N$ if the spin winding is an odd number. For even winding numbers, such as $N=2$, there is no CD. This can be understood by treating a $N=2$ skyrmion as two $N=1$ skyrmions pulled together ${ }^{2}$ (see Fig. 2b). These two $N=1$ skyrmions have opposite chirality, thus there is no global chirality of this spin configuration. As CD is sensitive to chiral structures ${ }^{38}$, the total CD for this state is zero. This also applies for other even winding number systems.

On the other hand, by varying the linear polarization $\beta$ of the incident light from $0^{\circ}$ to $180^{\circ}$, one can measure the polarizationdependent scattering intensity at each $\Psi$. By covering $\Psi$ in the range from $0^{\circ}$ to $360^{\circ}$, a PAM is obtained. The PAM plot in Fig. 2i-1 shows hump-like, two-dimensional peaks of equal height. The peaks appear around $\beta \approx 90^{\circ}$, and modulate along $\Psi$. The periodicity of the PAM signal is twice that of the winding number. This PAM feature is consistent with the analytical solution described by equation (3).

If both the CD and PAM data can be fitted by the two equations (3) and (4) simultaneously, the winding number can be unambiguously determined. If the detailed spin structure of the motif varies, such that the exact mapping from physical space to order parameter space changes within the same homotopy class, the corresponding shapes of the $\mathrm{CD}$ and PAM signals only undergo a linear shift, while the periodicities do not change (see Supplementary Note 1). Therefore, the topological robustness is also reflected in this type of X-ray scattering measurement.

Experimental demonstration. To demonstrate the measurement principle, we performed experiments on single-crystalline $\mathrm{Cu}_{2} \mathrm{OSeO}_{3}$ with the setup sketched in Fig. 1d. At $57 \mathrm{~K}$, and in an applied magnetic field of $32 \mathrm{mT}$, the skyrmion lattice phase emerges, manifesting itself as a hexagonal lattice of $N=1$ topological motifs. The lattice gives rise to the six-fold-symmetric diffraction pattern in reciprocal space, shown in Fig. 3a. The six sharp first-order magnetic peaks correspond to the 'unit cell' of the skyrmion lattice, with one of them locked along $h$, that is, the [100] crystallographic direction in real space. This is due 
a

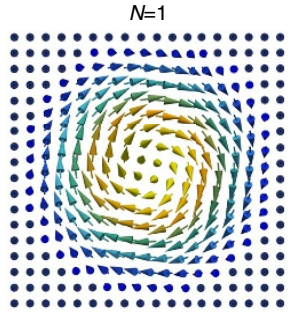

e

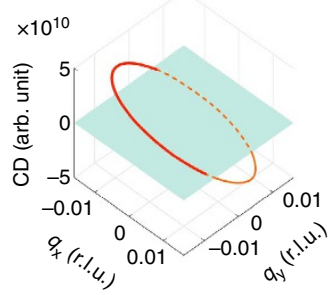

i

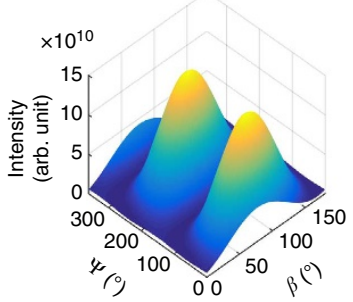

b

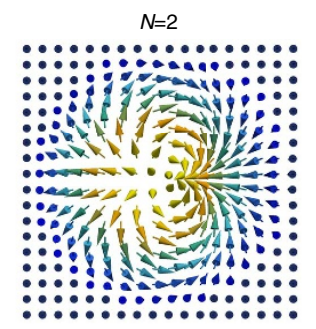

f

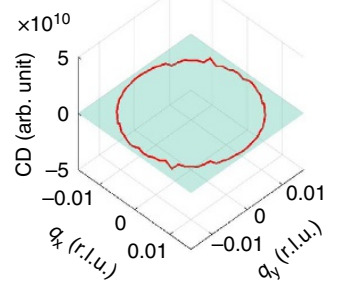

j

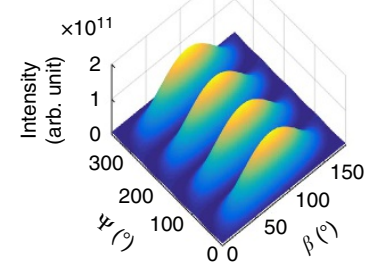

C

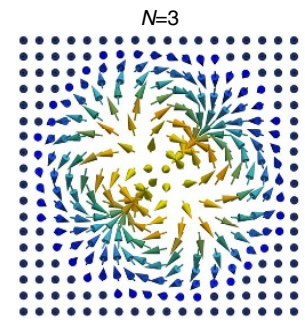

g

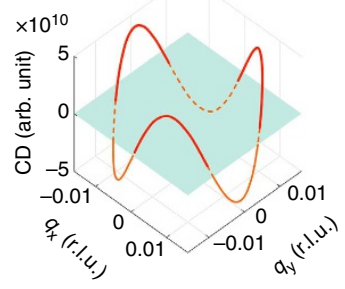

k

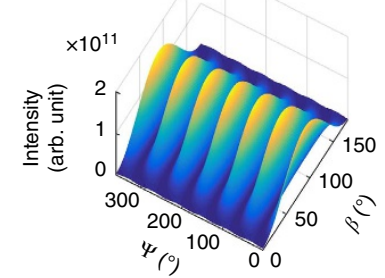

d

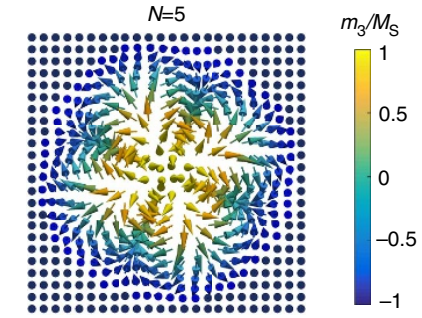

h

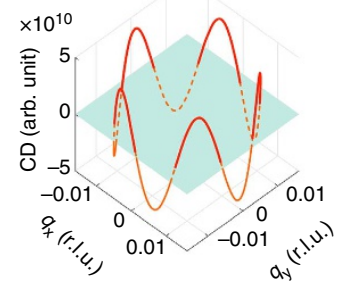

I

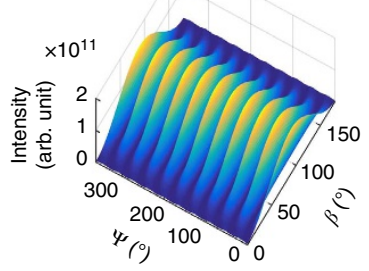

Figure 2 | Numerical determination of the topological winding number. (a-d) Spin configurations with topological winding numbers of 1, 2, 3 and 5, respectively. These spin textures are the motifs that generate the two-dimensional, periodically ordered lattices which can be measured by diffraction techniques. (e-h) CD cross-section as a function of $\Psi$ for topologically ordered systems. Here, $\Psi$ is represented by a loop (shown in red) in reciprocal space $\left(q_{x}^{\text {diff }}, q_{y}^{\text {diff }}\right.$ ), where $q_{x}^{\text {diff }}=q \cos \Psi, q_{y}^{\text {diff }}=q \sin \Psi$, and $q=0.0158$ r.l.u. is the absolute value of the skyrmion lattice modulation wavevector for $\mathrm{Cu}_{2} \mathrm{OSeO}_{3}$. The condition for vanishing $\mathrm{CD}(\mathrm{CD}=0)$ is indicated by the light blue plane. The $\mathrm{CD}$ signal is symmetric about this plane for integer winding numbers. The periodicity of the CD modulation equals to $N$. Note that for even $N$ the CD signal is zero. (i-I) PAMs. The calculations are performed by rotating $\beta$ from $0^{\circ}$ to $180^{\circ}$ at each $\Psi$, and by mapping out $\Psi$ from $0^{\circ}$ to $360^{\circ}$. The total number of humps is equivalent to $2 \mathrm{~N}$. Note that for integer winding numbers the humps are of equal height.

to the higher-order magnetic anisotropy of the material ${ }^{34,36,37}$. Note that the coordinates $\left(q_{x}, q_{y}\right)$ used here, as defined in Fig. 1d, are independent of the crystallographic directions. Therefore, by rotating $\Psi$, the same modulation wavevectors rotate accordingly in the coordinate system (see Fig. 3b,c). The measured CD intensity as a function of $\Psi$ (see Fig. 3d) shows exactly one period when the X-rays map the physical space once, suggesting that the skyrmion motif has a winding number of $N=1$. Moreover, as shown in Fig. 3e,f, the PAM is in excellent agreement with the theoretical calculations presented in Fig. $2 \mathrm{i}$. The equal height of the two humps confirms the $N=1$ topology of this material.

\section{Discussion}

Another type of $N=1$ system, which does not carry chirality, is the so-called Néel-type skyrmion ${ }^{33}$ (see Fig. 4a). Its spin texture has a different appearance; however, it is topologically equivalent to the other skyrmion form. Consequently, the $\mathrm{CD}$ profile in Fig. 4b shows the same periodicity; however, a constant phase shift, as compared with Fig. 2e, appears. The phase shift, on the other hand, is due to the different mapping of the spin configuration under a continuous transformation. The analytical solution takes the value of $\Phi_{1}=\pi / 2$ for equation (4). The same behaviour is found for the PAM, as shown in Fig. $4 \mathrm{c}$, for which the shape and height of the two humps are essentially the same as in Fig. 2i; however, the entire pattern undergoes a linear shift along $\Psi$. The analytical solution takes the value of $\Phi_{2}=\pi / 6$ for equation (3). Moreover, if the winding number is negative (see Fig. 4d), the CD signal still shows the same periodicity, that is, it does not distinguish between $N$ and $-N$. However, the appearance of the humps is fundamentally different (compare Fig. 4 f with Fig. 4c).

In summary, we have demonstrated that for a long-wavelength magnetically ordered system, the topological winding number of the motif can be unambiguously determined by polarized X-rays. First, our polarization-dependent REXS strategy is a direct measurement method as the winding number is naturally encoded in the underlying physics of the light-matter interaction, and is explicit in the measurement principle, expressed in equation (3) and (4). Second, although we used resonant soft X-ray diffraction for the demonstration of the measurement principle, the fundamentally same theory, with slight modifications, can be applied to the hard X-ray wavelength regime as well. It can further be expanded to non-resonant magnetic X-ray scattering by adding certain corrections. Third, this experimental technique can be applied to a wide range of magnetic systems, including both metallic and insulating materials, as well as other genres of materials that host topologically ordered spin systems, making it a general experimental principle. 
a

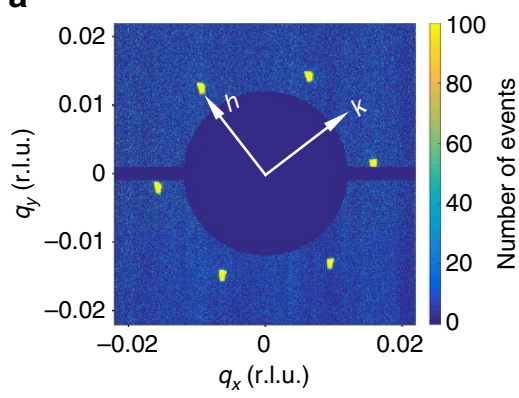

d

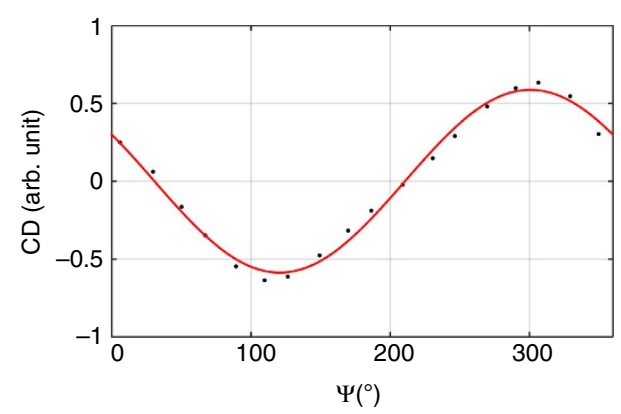

b

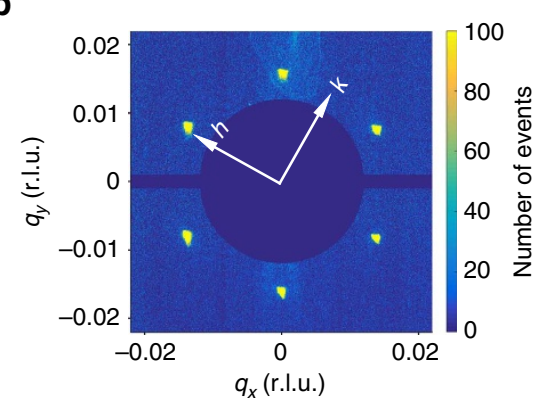

e

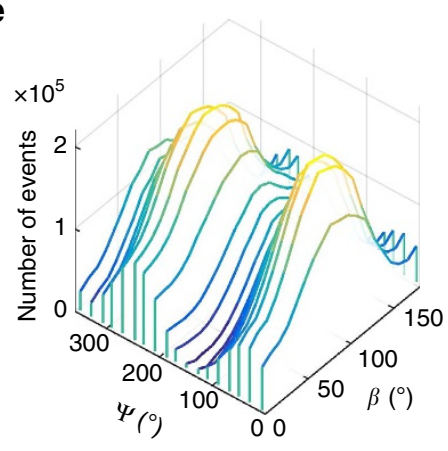

c

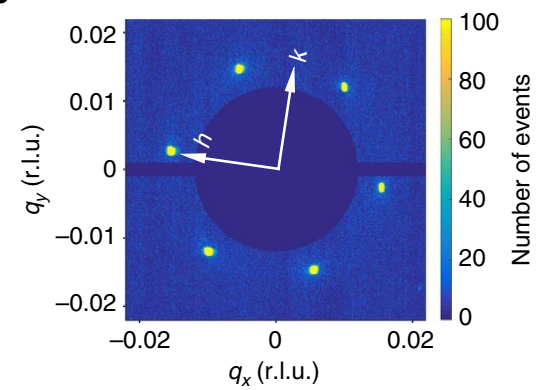

$\mathbf{f}$

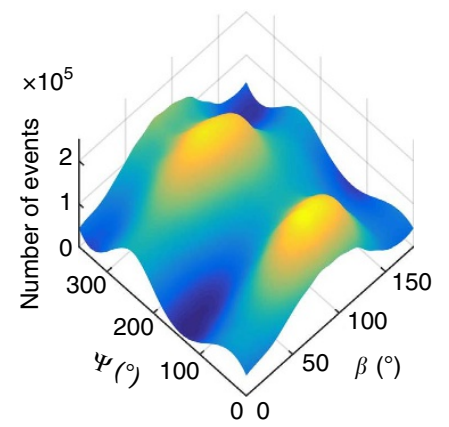

Figure 3 | Experimental determination of the topological winding number. (a-c) Resonant soft $\mathrm{X}$-ray magnetic diffraction from $\mathrm{Cu}_{2} \mathrm{OSeO}_{3}$ for three different azimuthal angles: $\Psi=5.95^{\circ}, 29.56^{\circ}$ and $50.17^{\circ}$, respectively. The photon energy is tuned to $931.25 \mathrm{eV}$ and the magnetic satellites are observed around the (001) structural peak. The figures show the reciprocal space maps within the ( $h k 1)$-plane, obtained by integrating the scattering intensities for left- and right-circularly polarized incident light. The coordinate system is defined in Fig. 1d. The temperature was $57 \mathrm{~K}$ and the applied magnetic field of $32 \mathrm{mT}$ along the $z$ direction, which is also parallel to the [001] crystallographic direction. (d) CD signal as a function of $\Psi$. The black dots are the measured data, while the red line is a fit using equation (3). (e) Measured PAM, and (f) interpolation obtained by fitting equation (4). The interpolated PAM shows excellent agreement with the calculated result shown in Fig. $2 \mathrm{i}$, in which two humps of equal height can be observed at $\beta \approx 90^{\circ}$.

\section{Methods}

Polarization-dependent resonant magnetic X-ray scattering. For the derivation of the polarization-dependent REXS signal of an $N$-skyrmion system, we start from the basic resonant X-ray scattering process from chiral magnets ${ }^{39-42}$. For a single magnetic ion at site $n$ carrying a moment $\mathbf{m}_{n}$ the resonant scattering form factor in the electric-dipole approximation takes the form:

$$
f_{n}=\tilde{\mathfrak{f}}_{0}\left(\epsilon_{\mathrm{s}}^{*} \cdot \epsilon_{\mathrm{i}}\right)-i \tilde{f}_{1}\left(\epsilon_{\mathrm{s}}^{*} \times \epsilon_{\mathrm{i}}\right) \cdot \mathbf{m}_{n},
$$

where $\epsilon_{\mathrm{i}}$ and $\epsilon_{\mathrm{s}}$ are the polarization unit vectors of the incident and outgoing $\mathrm{X}$-rays, and the asterisks denotes the complex conjugate. The $\tilde{f}_{0}$ and $\tilde{f}_{1}$ terms are the charge and the linear magnetic part of the energy-dependent resonance amplitude, respectively. The $\tilde{f}_{0}$ term describes the anomalous charge scattering at resonance, which is added to the Thomson scattering part. The $\mathfrak{f}_{1}$ term describes resonant magnetic scattering, which can be of the same order of magnitude as charge scattering. In equation (5), we have neglected the term that is quadratic in the magnetization as it is much smaller than the leading terms, and which gives rise to higher-order effects.

In the first Born approximation, the diffraction intensity for the scattering vector $\mathbf{Q}=\mathbf{k}_{\mathrm{s}}-\mathbf{k}_{\mathrm{i}}$ from a periodic lattice with sites $\mathbf{r}_{n}$ can be written as

$$
I(\mathbf{Q}) \propto\left|\sum_{n} f_{n} e^{i \mathbf{Q} \cdot \mathbf{r}_{n}}\right|^{2} .
$$

The complex amplitudes $\tilde{f}_{0}$ and $\mathfrak{f}_{1}$ are energy-dependent. Here we take them as constant, since in our numerical calculations the photon energy is not varied.

The coordinate system used to carry out the polarization-dependent study of the scattering cross-section is shown in Fig. 1b. The Cartesian coordinates are determined by the scattering plane (containing $\mathbf{Q}$ ), that is, the $x-z$-plane in this case. The $y$ axis is perpendicular to this plane. This defines the three components for the magnetization vectors, as well as the reciprocal space coordinates $\left(q_{x}, q_{y}, q_{z}\right)$. Thus, $\mathbf{k}_{\mathrm{i}}=k(\cos \alpha, 0, \sin \alpha), \mathbf{k}_{\mathrm{s}}=\mathrm{k}(\cos \alpha, 0,-\sin \alpha), \mathbf{k}_{\mathrm{s}} \times \mathbf{k}_{\mathrm{i}}=k(0,-2 \cos \alpha \sin \alpha, 0)$, where the magnitude of the X-ray wavevector, $k$, relates to the photon energy $[k=2 \pi / \lambda=E /(\hbar c)]$.

In the Poincaré-Stokes representation, the polarization of the incident X-rays is characterized by $\mathbf{P}=\left(P_{0}, P_{1}, P_{2}, P_{3}\right)$. For left- and right-circularly polarized light, $P_{0}=1, P_{1}=P_{2}=0, P_{3}= \pm 1$. For linearly polarized light, $P_{0}=1, P_{1}=\cos 2 \beta$, $P_{2}=\sin 2 \beta, P_{3}=0$.
For a magnetic system carrying incommensurate magnetic modulations, magnetic diffraction occurs as satellites $\mathbf{q}$ surrounding the structure peak $\mathbf{G}$, so that $\mathbf{Q}=\mathbf{G}+\mathbf{q}$. Therefore, the charge and magnetic part of the diffraction can be separated, and no charge-magnetic interference term is expected. Consequently, at the diffraction condition for the periodic magnetic structure, the scattering intensity is described by the magnetic part (that is, second term of equation (5)). It is straightforward, but rather tedious, to derive the intensity for the magnetic scattering, which is given by ${ }^{41}$ :

$$
\begin{aligned}
I(\mathbf{Q}) & =\frac{1}{2}\left|F_{1}\right|^{2}\left(P_{0}+P_{1}\right)\left|\mathbf{k}_{\mathrm{s}} \cdot \mathbf{M}(\mathbf{Q})\right|^{2} \\
& +\frac{1}{2}\left|F_{1}\right|^{2}\left(P_{0}-P_{1}\right)\left[\left|\mathbf{k}_{\mathrm{i}} \cdot \mathbf{M}(\mathbf{Q})\right|^{2}+\left|\left(\mathbf{k}_{\mathrm{s}} \times \mathbf{k}_{\mathrm{i}}\right) \cdot \mathbf{M}(\mathbf{Q})\right|^{2}\right] \\
& -\left|F_{1}\right|^{2} \operatorname{Re}\left[\left(P_{2}+i P_{3}\right)\left(\mathbf{k}_{\mathrm{s}} \cdot \mathbf{M}^{*}(\mathbf{Q})\right)\left(\mathbf{k}_{\mathrm{s}} \times \mathbf{k}_{\mathrm{i}}\right) \cdot \mathbf{M}(\mathbf{Q})\right],
\end{aligned}
$$

where $F_{1}$ is the energy-dependent resonant term, and $\mathbf{M}(\mathbf{Q})$ is the Fourier transform of the real-space magnetic moment modulation $\mathbf{m}(\mathbf{r})$ at $\mathbf{Q}$. Note that $\mathbf{k}_{\mathbf{i}}$ and $\mathbf{k}_{\mathrm{s}}$ are tuned to fulfil the diffraction condition for $\mathbf{Q}$.

X-ray polarization dependence of the winding number. As shown in Fig. 1a, a one-dimensional proper-screw helix pitch ${ }^{43}$, otherwise called 'Bloch-type' helix, propagating along $x$, can be written as

$$
\begin{aligned}
& m_{1}=0, \\
& m_{2}=M_{\mathrm{S}} \cos \left(\mathbf{q}_{\mathrm{h}} \cdot \mathbf{r}\right), \\
& m_{3}=M_{\mathrm{S}} \sin \left(\mathbf{q}_{\mathrm{h}} \cdot \mathbf{r}\right),
\end{aligned}
$$

where $\mathbf{q}_{\mathrm{h}}$ is the helix propagation wavevector. We define a base position of the helix such that $\mathbf{q}_{\mathrm{h}}$ is along the $x$ axis, this also corresponds to $\Psi=0^{\circ}$. While the X-rays probe the physical space at an finite angle $\Psi$, the order parameter space

magnetization profile rotates the base helix position specified by equation (8) by $N \Psi$ within the $q_{x}-q_{y}$-plane. By applying the rotation matrix

$\mathcal{R}_{N \Psi}=\left(\begin{array}{ccc}\cos (N \Psi) & -\sin (N \Psi) & 0 \\ \sin (N \Psi) & \cos (N \Psi) & 0 \\ 0 & 0 & 1\end{array}\right)$ to equation (8), the rotated magnetic

structure becomes

$$
\begin{aligned}
& m_{1}=-M_{\mathrm{S}} \sin (N \Psi) \cos \left(\mathbf{q}_{\mathrm{h}} \cdot \mathbf{r}\right), \\
& m_{2}=M_{\mathrm{S}} \cos (N \Psi) \cos \left(\mathbf{q}_{\mathrm{h}} \cdot \mathbf{r}\right), \\
& m_{3}=M_{\mathrm{S}} \sin \left(\mathbf{q}_{\mathrm{h}} \cdot \mathbf{r}\right) .
\end{aligned}
$$


a

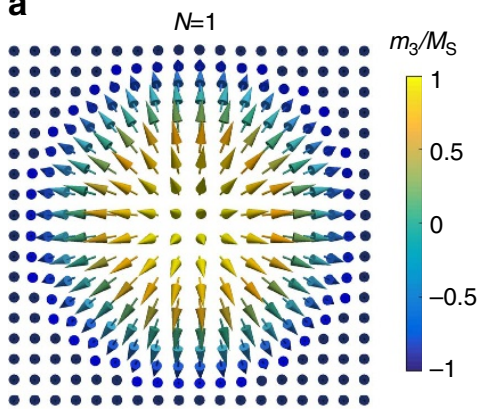

d

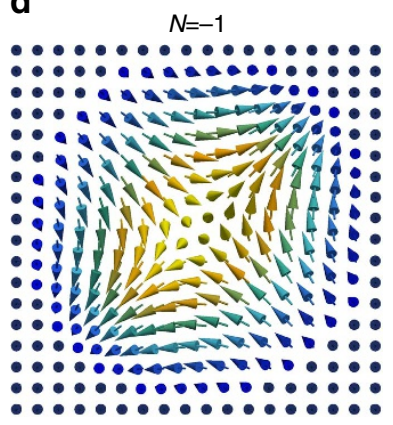

b

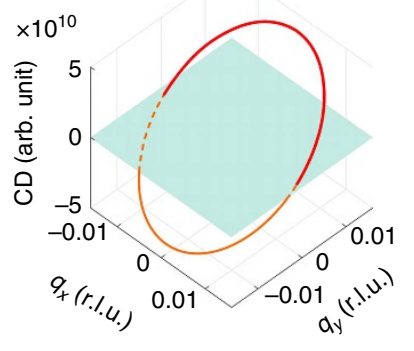

e

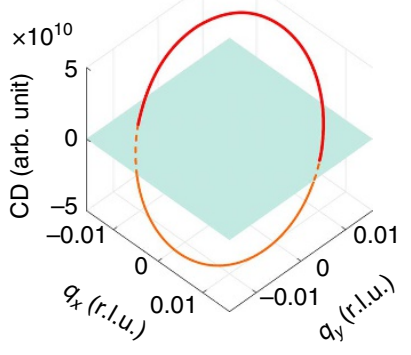

C

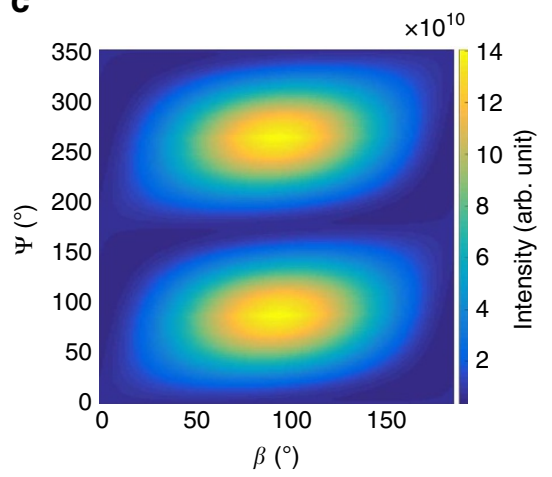

$\mathbf{f}$

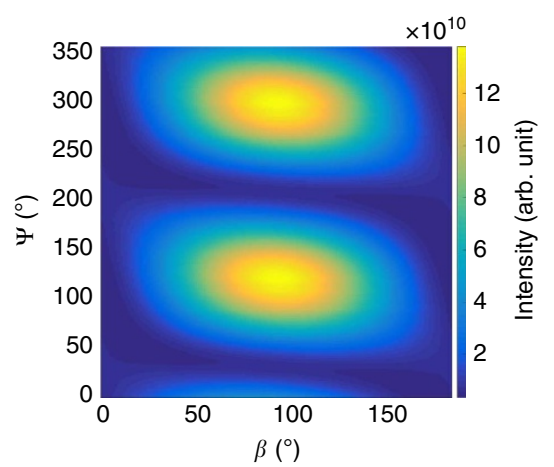

Figure 4 | Robustness of the measurement principle. (a) Spin configuration of a Néel-type skyrmion with $N=1$, (b) calculated CD profile, and, (c) calculated PAM. (d) Spin configuration of an anti-skyrmion with $N=-1$. Note that the anti-skyrmion, as well as other topological entities with negative topological numbers, are not energetically stable states. Nevertheless, our experimental principle can be applied, as shown in e,f in which the CD profile and PAM periodicity suggest the correct absolute value of the winding number. Moreover, negative winding numbers give rise to a fundamentally different PAM shape of the humps. This provides an additional way to distinguish positive from negative values of $N$.

To meet the diffraction condition for $\mathbf{Q}=\mathbf{G}+\mathbf{q}_{\mathrm{b}}$ at $\Psi$, one has to bring $\mathbf{Q}$ into the scattering plane. In a common four-circle diffractometer, this is achieved by compensating the diffraction offset with the other two rotation axes, that is, the $\alpha$ axis, and the $\kappa$ axis, which is perpendicular to both the $\alpha$ and $\Psi$ axes. As a consequence, the components of the magnetic structure transform into:

$$
\left(\begin{array}{c}
m_{1}^{\prime} \\
m_{2}^{\prime} \\
m_{3}^{\prime}
\end{array}\right)=\mathcal{R}_{\alpha} \mathcal{R}_{\kappa}\left(\begin{array}{l}
m_{1} \\
m_{2} \\
m_{3}
\end{array}\right)
$$

where $\mathcal{R}_{\kappa}$ and $\mathcal{R}_{\alpha}$ are the rotation matrices corresponding to the $\kappa$ and $\alpha$ rotation axes, and the combination of the two rotations brings $\mathbf{Q}$ into the scattering plane for the diffraction condition.

However, it is essential to note that this change would be negligible for most of the long-wavelength modulated magnetic structures. For example, $\mathrm{Cu}_{2} \mathrm{OSeO}_{3}$ has $q a=0.0158$ (ref. 37), where $a$ is the lattice constant. Therefore, for $\mathbf{G}=(0,0,1), \mathbf{Q}=\mathbf{G}+\mathbf{q}_{\mathrm{h}}$, the change of $\alpha$ is less than $0.9^{\circ}$ for all $\Psi$ angles. This makes $\mathcal{R}_{\kappa}$ and $\mathcal{R}_{\alpha} \approx 1$. Therefore, the long-wavelength approximation suggests $\left(\begin{array}{l}m_{1}^{\prime} \\ m_{2}^{\prime} \\ m_{3}^{\prime}\end{array}\right) \approx\left(\begin{array}{l}m_{1} \\ m_{2} \\ m_{3}\end{array}\right)$, as well as $\mathbf{M}(\mathbf{Q}) \approx \mathbf{M}\left(\mathbf{q}_{\mathrm{h}}\right)$, and we can take one angle $\alpha$ for the diffraction condition of all $\Psi$ positions.

Thus, the Fourier transform of equation (9) at the diffraction condition of $\mathbf{q}_{\mathrm{h}}$ takes the form

$$
\begin{aligned}
& M_{1}\left(\mathbf{q}_{\mathrm{h}}, \Psi\right)=-\pi M_{\mathrm{S}} \sin (N \Psi), \\
& M_{2}\left(\mathbf{q}_{\mathrm{h}}, \Psi\right)=\pi M_{\mathrm{S}} \cos (N \Psi), \\
& M_{2}\left(\mathbf{q}_{\mathrm{h}}, \Psi\right)=-i \pi M_{\mathrm{S}} .
\end{aligned}
$$

Inserting equation (11) into equation (7), and evaluating the expressions described above, the $\mathrm{CD}$ profile is obtained as

$$
I_{\mathrm{CD}}=4 Y \sin ^{2} \alpha \cos \alpha \cos (N \Psi)
$$

where $Y=\pi^{2}\left|F_{1}\right|^{2} k^{2} M_{S}^{2}$. However, note that the $\mathrm{CD}$ intensity is zero for even values of $N$. This condition is not captured by the analytical relationship of equation (12); however, it can be generalized from the numerical calculations.

The reason why the $\mathrm{CD}$ vanishes is discussed in the main text, and is based on the assumption that, for an example, an $N=2$ skyrmion can be considered as two $N=1$ skyrmions pulled together ${ }^{2}$. The 'chirality cancelling' effect does not occur for odd winding number motifs; however, it exists for all even winding numbers. Moreover, as will be discussed shortly, adding another phase factor $\Phi_{1}$ is also necessary for generalizing the $\mathrm{CD}$ relationship to $N$-skyrmions.

The linear polarization dependence can be derived as

$$
\begin{aligned}
I & =Y \sin ^{2} \alpha+Y \cos ^{2} \alpha \sin ^{2}(N \Psi) \\
& +4 Y \cos ^{2} \alpha \sin ^{2} \alpha \cos ^{2}(N \Psi) \sin ^{2} \beta \\
& -Y \cos ^{2} \alpha \sin \alpha \sin (2 N \Psi) \sin 2 \beta
\end{aligned}
$$

Equations (12) and (13) are the foundation of our measurement principle, and are derived based on a standard one-dimensional helix structure. Therefore, this analytical form is only valid for 'standard' types of spin configurations, for an example, $N=1$ chiral skyrmions with $\chi= \pm \pi / 2$. However, in principle, there is an infinite number of homotopies for a certain winding number, that is, the same topological property will always hold if continuous transformations are acting on a 'standard' skyrmion configuration, as we have used and derived so far. For example, if the one-dimensional helix takes other forms, such as a cycloidal type structure $^{33}$, the overall spin texture will change while the winding number remains invariant. As shown in the Supplementary Note 1, this degree of freedom is dealt with by adding a phase factor to equations (12) and (13), which makes the measurement principle generally valid for all cases.

Numerical calculations. Numerical calculations were carried out using the materials parameters of $\mathrm{Cu}_{2} \mathrm{OSeO}_{3}$, that is, a helix pitch of $60 \mathrm{~nm}$. This leads to a skyrmion core-to-core distance of $\sim 69.28 \mathrm{~nm}$, as well as a wavevector of $\sim 0.015$ r.l.u. Resonant X-ray scattering at the $\mathrm{Cu} L_{3}$ edge with a photon energy of $931.25 \mathrm{eV}$ gives $k=2 \pi \times 4.7187 \mathrm{~nm}^{-1}$, with $\alpha \approx 48.24^{\circ}$ for the $(0,0,1)$ diffraction peak. In the calculation, $F_{1}$ and $M_{\mathrm{S}}$ are kept constant as the $\mathrm{CD}$ profile and PAM are measured for the same photon energy and temperature. For detailed numerical results, please see Supplementary Note 1.

REXS. Resonant soft X-ray scattering experiments were carried out in the RASOR diffractometer on beamline I10 at the Diamond Light Source (UK). Single crystals of $\mathrm{Cu}_{2} \mathrm{OSeO}_{3}$ are pre-characterized by X-ray diffraction and electron back-scattering diffraction to confirm the crystalline quality and single chirality. Magnetometry measurements were performed to map out the magnetic phase diagram. The polished crystal surface was (001)-oriented for the subsequent resonant X-ray scattering measurements. 
The incident soft X-ray beam with variable polarization was tuned to the $\mathrm{Cu} L_{3}$ edge. The experimental geometry is shown in Fig. 1d. The scattered beam is captured by either a CCD camera or a photodiode point detector. The modulated magnetic structure leads to satellites surrounding the structural peaks in reciprocal space. Further details about the experimental methods on resonant soft X-ray scattering can be found in refs 37,42. Polarization-dependent measurements are performed by varying the incident light polarization, while measuring the scattering intensities for different diffraction conditions for varying $\Psi$.

Data availability. The data that support the findings of this study are available from the corresponding author on request.

\section{References}

1. Nakahara, M. Geometry, Topology and Physics (IOP Publishing, 2003).

2. Mermin, N. D. The topological theory of defects in ordered media. Rev. Mod. Phys. 51, 591-648 (1979).

3. Braun, H.-B. Topological effects in nanomagnetism: from superparamagnetism to chiral quantum solitons. Adv. Phys. 61, 1-116 (2012).

4. Nagaosa, N. \& Tokura, Y. Topological properties and dynamics of magnetic skyrmions. Nat. Nanotechnol. 8, 899-911 (2013).

5. Garst, M. in Topological Structures in Ferroic Materials: Domain Walls, Vortices and Skyrmions 29-53 (Springer International Publishing, 2016).

6. Seki, S. \& Mochizuki, M. Skyrmions in Magnetic Materials (Springer, 2016).

7. Taguchi, Y., Oohara, Y., Yoshizawa, H., Nagaosa, N. \& Tokura, Y. Spin chirality, Berry phase, and anomalous Hall effect in a frustrated ferromagnet. Science 291, 2573-2576 (2001).

8. Haldane, F. D. M. Berry curvature on the fermi surface: anomalous Hall effect as a topological fermi-liquid property. Phys. Rev. Lett. 93, 206602 (2004).

9. Neubauer, A. et al. Topological hall effect in the a phase of MnSi. Phys. Rev Lett. 102, 186602 (2009).

10. Everschor, K., Garst, M., Duine, R. A. \& Rosch, A. Current-induced rotational torques in the skyrmion lattice phase of chiral magnets. Phys. Rev. B 84, 064401 (2011).

11. Jonietz, F. et al. Spin transfer torques in $\mathrm{MnSi}$ at ultralow current densities. Science 330, 1648-1651 (2010).

12. Everschor, K. et al. Rotating skyrmion lattices by spin torques and field or temperature gradients. Phys. Rev. B 86, 054432 (2012).

13. Yu, X. Z. et al. Skyrmion flow near room temperature in an ultralow current density. Nat. Commun. 3, 988 (2012).

14. Volovik, G. E. Linear momentum in ferromagnets. J. Phys. C: Solid State Phys. 20, L83-L88 (1987).

15. Zang, J., Mostovoy, M., Han, J. H. \& Nagaosa, N. Dynamics of skyrmion crystals in metallic thin films. Phys. Rev. Lett. 107, 136804 (2011).

16. Schulz, T. et al. Emergent electrodynamics of skyrmions in a chiral magnet. Nat. Phys. 8, 301-304 (2012).

17. Mochizuki, M. Spin-wave modes and their intense excitation effects in skyrmion crystals. Phys. Rev. Lett. 108, 017601 (2012).

18. Onose, Y., Okamura, Y., Seki, S., Ishiwata, S. \& Tokura, Y. Observation of magnetic excitations of skyrmion crystal in a helimagnetic insulator $\mathrm{Cu}_{2} \mathrm{OSeO}_{3}$. Phys. Rev. Lett. 109, 037603 (2012).

19. Schwarze, T. et al. Universal helimagnon and skyrmion excitations in metallic, semiconducting and insulating chiral magnets. Nat. Mater. 14, 478-483 (2015).

20. Nagaosa, N. \& Tokura, Y. Emergent electromagnetism in solids. Phys. Scr T146, 014020 (2012)

21. Büttner, F. et al. Dynamics and inertia of skyrmionic spin structures Nat. Phys. 11, 225-228 (2015).

22. Jiang, W. et al. Blowing magnetic skyrmion bubbles. Science 349, 283-286 (2015).

23. Boulle, O. et al. Room-temperature chiral magnetic skyrmions in ultrathin magnetic nanostructures. Nat. Nanotechnol. 11, 449-454 (2016).

24. Woo, S. et al. Observation of room-temperature magnetic skyrmions and their current-driven dynamics in ultrathin metallic ferromagnets. Nat. Mater. 15, 501-506 (2016)

25. Yu, X. Z. et al. Biskyrmion states and their current-driven motion in a layered manganite. Nat. Commun. 5, 3198 (2014).

26. Wang, W. et al. A centrosymmetric hexagonal magnet with superstable biskyrmion magnetic nanodomains in a wide temperature range of 100-340 K. Adv. Mater. 28, 6887-6893 (2016).

27. Yu, X. Z. et al. Real-space observation of a two-dimensional skyrmion crystal. Nature 465, 901-904 (2010)

28. Heinze, S. et al. Spontaneous atomic-scale magnetic skyrmion lattice in two dimensions,. Nat. Phys 7, 713-718 (2011).

29. Milde, P. et al. Unwinding of a skyrmion lattice by magnetic monopoles. Science 340, 1076-1080 (2013).
30. Monchesky, T. L., Loudon, J. C., Robertson, M. D. \& Bogdanov, A. N. Comment on 'Robust formation of skyrmions and topological Hall effect anomaly in epitaxial thin films of MnSi. Phys. Rev. Lett. 112, 059701 (2014).

31. Meynell, S. A. et al. Hall effect and transmission electron microscopy of epitaxial MnSi thin films. Phys. Rev. B 90, 224419 (2014).

32. Bogdanov, N. \& Yablonskii, D. A. Thermodynamically stable 'vortices' in magnetically ordered crystals. The mixed state of magnets. Sov. Phys. JETP 68, 101-103 (1989).

33. Kézsmárki, I. et al. Neel-type skyrmion lattice with confined orientation in the polar magnetic semiconductor $\mathrm{GaV}_{4} \mathrm{~S}_{8}$. Nat. Mater. 14, 1116-1122 (2015).

34. Adams, T. et al. Long-wavelength helimagnetic order and skyrmion lattice phase in $\mathrm{Cu}_{2} \mathrm{OSeO}_{3}$. Phys. Rev. Lett. 108, 237204 (2012).

35. Seki, S., Yu, X. Z., Ishiwata, S. \& Tokura, Y. Observation of skyrmions in a multiferroic material. Science 336, 198-201 (2012).

36. Langner, M. C. et al. Coupled Skyrmion sublattices in $\mathrm{Cu}_{2} \mathrm{OSeO}_{3}$. Phys. Rev. Lett. 112, 167202 (2014)

37. Zhang, S. L. et al. Multidomain skyrmion lattice state in $\mathrm{Cu}_{2} \mathrm{OSeO}_{3}$. Nano Lett. 16, 3285-3291 (2016).

38. Dürr, H. A. et al. Chiral magnetic domain structures in ultrathin FePd films. Science 284, 2166-2168 (1999).

39. Hannon, J. P., Trammell, G. T., Blume, M. \& Gibbs, D. X-ray resonance exchange scattering. Phys. Rev. Lett. 61, 1245-1248 (1988).

40. Blume, M. \& Gibbs, D. Polarization dependence of magnetic x-ray scattering Phys. Rev. B 37, 1779-1789 (1988).

41. van der Laan, G. Soft x-ray resonant magnetic scattering of magnetic nanostructures. C. R.Phys. 9, 570-584 (2008).

42. Zhang, S. L. et al. Resonant elastic x-ray scattering from the skyrmion lattice in $\mathrm{Cu}_{2} \mathrm{OSeO}_{3}$. Phys. Rev. B 93, 214420 (2016).

43. Tokura, Y. \& Seki, S. Multiferroics with spiral spin orders. Adv. Mater. 22, 1554-1565 (2010).

\section{Acknowledgements}

The resonant soft X-ray scattering experiments were carried out on beamline I10 at the Diamond Light Source, UK, under proposals SI-11784 and SI-12958. S.L.Z. and T.H. acknowledge financial support by the Semiconductor Research Corporation (SRC) and EPSRC (EP/N032128/1). We thank H. Berger for providing the crystal and A. Bauer and C. Pfleiderer for fruitful discussions.

\section{Author contributions}

S.L.Z. conceived and derived polarization-dependent REXS for the determination of the winding number. S.L.Z., T.H. and G.v.d.L. designed and performed the experiments, as well as carried out the data analysis. S.L.Z. performed the numerical calculations. S.L.Z. G.v.d.L. and T.H. discussed all aspects of the work and wrote the manuscript.

\section{Additional information}

Supplementary Information accompanies this paper at http://www.nature.com/ naturecommunications

Competing financial interests: The authors declare no competing financial interests.

Reprints and permission information is available online at http://npg.nature.com/ reprintsandpermissions/

How to cite this article: Zhang, S. L. et al. Direct experimental determination of the topological winding number of skyrmions in $\mathrm{Cu}_{2} \mathrm{OSeO}_{3}$. Nat. Commun. 8, 14619 doi: $10.1038 /$ ncomms14619 (2017).

Publisher's note: Springer Nature remains neutral with regard to jurisdictional claims in published maps and institutional affiliations.

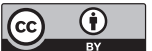

This work is licensed under a Creative Commons Attribution 4.0 International License. The images or other third party material in this article are included in the article's Creative Commons license, unless indicated otherwise in the credit line; if the material is not included under the Creative Commons license, users will need to obtain permission from the license holder to reproduce the material To view a copy of this license, visit http://creativecommons.org/licenses/by/4.0/

C The Author(s) 2017 